\title{
CBT added to medical management improved clinical outcomes in rheumatoid arthritis
}

Sharpe L, Sensky T, Timberlake N, et al. A blind, randomized, controlled trial of cognitive-behavioural intervention for patients with recent onset rheumatoid arthritis: preventing psychological and physical morbidity. Pain 2001 Jan;89:275-83.

QUESTION: Does the addition of cognitive behavioural therapy (CBT) to medical management improve clinical outcomes in patients with rheumatoid arthritis (RA)?

\section{Design}

Randomised \{allocation concealed*\}†, blinded (outcome assessor)*, controlled trial with 6 months of follow up.

\section{Setting}

3 hospital rheumatology clinics in or near London, UK.

\section{Patients}

56 patients who were $18-75$ years of age, had had definite or classic RA for $<2$ years, and tested seropositive for RA. Exclusion criteria were history of mental illness or alcohol or drug abuse, or insufficient fluency in English. Follow up was $80 \%$ (mean age 55 y, $70 \%$ women).

\section{Intervention}

Patients were allocated to routine medical management and CBT $(n=23)$ or routine medical management alone $(\mathrm{n}=22) .2$ psychologists provided CBT according to a treatment manual during eight 1 hour sessions over 8 weeks, which included an educational component plus self management skills (relaxation training, attention diversion, goal setting, pacing, problem solving, cognitive restructuring, assertiveness and communication, and management of flare ups or high risk situations).

\section{Main outcome measures}

Anxiety and depression (Hospital Anxiety and Depression Scales [HADS]), pain (self reports), disability (Health Assessment Questionnaire), joint inflammation (Ritchie Articular Index), and disease measures (erythrocyte sedimentation rate and $\mathrm{C}$ reactive protein concentration).

\section{Main results}

At follow up, patients in the CBT group were less depressed than at baseline, whereas those in the control group became more depressed (mean HADS depression score change $-0.8 v 1.4, \mathrm{p}=0.02)$. More patients in the CBT group than in the control group had improved joint inflammation scores at 6 months $(\mathrm{p}<0.05)$ (table). The groups did not differ for anxiety, pain ratings, disability ratings, or disease measures.

\section{Conclusion}

The addition of cognitive behavioural therapy to medical management reduced depression and joint inflammation at 6 months in patients with rheumatoid arthritis.

*See glossary.

†Information provided by author.

Medical management plus cognitive behavioural therapy (CBT) v medical management alone (control) for patients with RA

\begin{tabular}{|c|c|c|c|c|}
\hline Outcomes at 6 months & CBT & Control & $\operatorname{RRR}(95 \% \mathrm{Cl})$ & NNT (Cl) \\
\hline No change in RAI score & $48 \%$ & $55 \%$ & $12 \%(-56$ to 51$)$ & Not significant \\
\hline$\geqslant 25 \%$ improvement in symptoms (RAl score) & $48 \%$ & $18 \%$ & $163 \%(6$ to 605$)$ & $4(2$ to 55$)$ \\
\hline
\end{tabular}

\begin{tabular}{l}
\hline website extra \\
\hline Additional references \\
appear on the \\
Evidence-Based \\
Mental Health \\
website \\
\hline www.ebmentalhealth. \\
com
\end{tabular}

www.ebn

\section{COMMENTARY}

Evidence that psychological interventions can assist the course of certain medical treatments is increasing. ${ }^{1}$ Most often, the changes are in psychological dimensions that enhance participation in or response to medical interventions. ${ }^{1}$ Differences in research design, and in the type and timing of intervention, limit the clinical applicability of study findings. ${ }^{2}$

Sharpe $e t$ al add further support to the finding that CBT can reduce depressive symptoms in people with chronic illness, despite it often worsening in those patients without intervention. These changes persisted at 6 months follow up. The treatment group improved physically compared with the control group. This is an important contribution to the RA literature and to the position that psychological interventions in medicine affect physical functioning and can help treat active disease.

Perhaps the most important contribution is the evaluation of the timing of intervention: earlier interventions can be an effective adjunct to early post diagnostic medical treatment, and may have greater and perhaps more lasting effects than later interventions. The findings support the effectiveness of CBT as an adjunct to medical care early in the course of disease, but the study design does not test whether efficacy is enhanced by the earlier intervention.

The authors note that an alternative hypothesis to the effect of the CBT is that therapist attention, independent of intervention content, contributed to the changes. The authors acknowledge this possibility but noted that the addition of an attention control group was not feasible because of statistical power considerations. They also observed that people in the $15 \%$ dropout pool were younger, more impaired, and had more active disease. For that subgroup, it cannot be concluded that the intervention has the same potential for effect.

This well designed study supports the use of CBT early in the disease cycle of patients with RA. Before we regularly adopt such interventions in clinical practice, however, further research comparing earlier with later interventions is necessary, and the efficacy of the intervention needs to be evaluated in younger and more impaired people.

Rodger Kessler, $\mathrm{PhD}$ Central Vermont Medical Center; Barre, Vermont, USA

1 Chiles J, Lambert M, Hatch A. The impact of psychological interventions on medical cost offset: a meta-analytic review. Clinical Psychology: Science and Practice 1999;6:204-20

2 Kessler R, Dane JR. Psychological and hypnotic preparation for anesthesia and surgery: an individual differences perspective. Int J Clin Exp Hypn 1996;44:189-207. 